\title{
Die Bearbeiter der 5. Auflage
}

Professor Dr. Hans-Jürgen Ahrens, Universität Osnabrück, Richter am OLG Celle a.D.

Professor Dr. Dorothea Assmann, Universität Potsdam

Dr. David-Christoph Bittmann, Vorsitzender Richter am LG Kaiserslautern

Professor Dr. Wolfgang Büscher, Vorsitzender Richter am Bundesgerichtshof a.D., Honorarprofessor

Universität Osnabrück

Professor Dr. Florian Eichel, Universität Bern

Univ.-Professor Dr. Thomas Garber, Karl-Franzens-Universität Graz

Professor Dr. Martin Gebauer, Universität Tübingen, Richter am OLG Stuttgart

Uwe Gerken, Vorsitzender Richter am OLG Oldenburg a.D.

Professor, Dr. Helge Großerichter, Rechtsanwalt, Honorarprofessor Ludwig-Maximilians-Universität, München

Dr. Sabine Hartmann, Rechtsanwältin in Kiel

Jens Joachim Haubold, Rechtsanwalt, Stuttgart

Professor Dr. Stefan Huber, LL.M., Universität Tübingen

Sabine Hufschmidt, Rechtsanwältin/Mediatorin (zertifiziert), Universität Potsdam

Professor Dr. Volker Michael Jänich, Universität Jena, Richter am OLG Jena

Professor Dr. Christoph A. Kern, LL.M. (Harvard), Universität Heidelberg, Direktor des Instituts für ausländisches und internationales Privat- und Wirtschaftsrecht

Dr. Ferdinand Kruis, Rechtsanwalt, München

PD, Dr. Florian Loyal, Universität Tübingen

Professor Dr. Wolfgang Lüke, LL.M. (Chicago), Universität Leipzig, Richter am OLG Dresden a.D.

Professor Dr. Katharina Lugani, Heinrich-Heine-Universität Düsseldorf

PD, Dr. Robert Magnus, Akademischer Rat a.Z., Universität Heidelberg

Professor Dr. Heinz-Peter Mansel, Universität Köln, Direktor des Instituts für internationales und ausländisches Privatrecht

Univ.-Professor Dr. Matthias Neumayr, Senatspräsident des OGH, Universität Salzburg

Dr. Carl Friedrich Nordmeier, Richter am LG Frankfurt am Main

Professor Dr. Christoph G. Paulus, LL.M. (Berkeley), Humboldt-Universität zu Berlin

Professor Dr. Dr. h.c. mult. Hanns Prütting, Universität zu Köln, Direktor des Instituts für Verfahrensrecht (1986-2016)

Dr. Hartmut Rensen, Richter am Bundesgerichtshof

Dr. Fabian Reuschle, Richter am LG Stuttgart

Professor Dr. Mathias Rohe, M.A., Universität Erlangen, Richter am OLG Nürnberg a.D.

Dr. Anastasia Schreiber, LL.M., Rechtsanwältin in Dortmund

PD, Dr. Christoph Schreiber, Akademischer Rat, Universität Erlangen-Nürnberg

Professor Dr. Dr. h.c. Rolf A. Schütze, Rechtsanwalt, Stuttgart, Honorarprofessor Universität Tübingen

Professor Dr. Götz Schulze, Universität Potsdam †

Professor Dr. Stefan Smid, Universität Kiel

Dr. Frank Spohnheimer, Akademischer Rat, Leibniz Universität Hannover

Professor Dr. Felipe Temming, LL.M. (LSE), Leibniz Universität Hannover

Professor Dr. Christoph Thole, Universität zu Köln, Direktor des Instituts für Verfahrensrecht und Insolvenzrecht

Professor Dr. Roderich C. Thümmel, LL.M. (Harvard), Rechtsanwalt, Stuttgart, Honorarprofessor Universität Tübingen

Dr. Eyk Ueberschär, Referent und Justitiar, Lehrbeauftragter, Universität Potsdam

Professor Dr. Barbara Völzmann-Stickelbrock, FernUniversität Hagen

Dr. Andreas Wax, Maître en Droit, Rechtsanwalt, Stuttgart

Professor Dr. Matthias Weller, Mag. rer. publ., Universität Bonn

PD, Dr. Matthias Wendland, LL.M. (Harvard), Ludwig-Maximilians-Universität München

Professor Dr. Stephan Weth, Universität des Saarlandes

Dr. Thomas Winter, Rechtsanwalt beim BGH

Dr. Wolfgang Winter, Rechtsanwalt, München

Professor Dr. Mark Zeuner, Rechtsanwalt/Insolvenzverwalter Hamburg, Honorarprofessor Universität Kiel 
